\title{
Uma proposta de estudo comparativo de NB-IoT vs LoRaWAN para aplicação em redes IIoT privadas para automação e monitoramento de processos
}

\author{
Danilo Farias de Carvalho ${ }^{1}$, Charles Christian Miers ${ }^{1}$ \\ ${ }^{1}$ Programa de Pós-Graduação em Computação Aplicada (PPGCAP) \\ Universidade do Estado de Santa Catarina (UDESC) \\ danilo.carvalhodedu.udesc.br - charles.miers@udesc.br
}

\begin{abstract}
Resumo. A Internet of Things (IoT) está cada vez mais pervasiva e ubíqua, nas mais variadas áreas. Diversas indústrias também estão incorporando esta inteligência aos seus processos através de Industrial Internet of Things (IIoT). Contudo, há diversas abordagens à implementação, sendo que as abordagens sem fios são as mais empregadas pela dinamicidade de implantação e gerenciamento. Este trabalho faz um estudo comparativo de duas das principais tecnologias sem fio que podem ser empregadas em redes de IIoT privadas (NB-IoT e LoRaWAN) no contexto de automação e monitoramento de processos com base em dados de um estudo caso real. Uma pesquisa referenciada correlaciona os requisitos de IIoT em função das características das redes NB-IoT e LoRaWAN.
\end{abstract}

\section{Introdução}

A automação de processos/industrial é uma área conhecida por ser conservadora [Wollschlaeger et al., 2017], e as vezes até mesmo reativa na utilização tecnologias de TI. Porém, o conservadorismo impacta financeiramente, ou com uma complexa infraestrutura. Nos últimos anos, a Tecnologia e Informação (TI) e a Tecnologia e Automação (TA) vem convergindo de tal forma, que muitas vezes fazem parte de uma mesma estrutura, podendo compartilhar alguns recursos mais físicos [Martins, 2015] e [Strey et al., 2017]. Com o advento da Indústria 4.0, uma maior integração tecnológica entre os processos é proposta, na qual é possível haver sensores e atuadores mais inteligentes com o apoio da IIoT [Sauter et al., 2011]. Além disso, com a tecnologia de redes móveis de Quinta Geração (5G) tende a intensificar-se, novas possibilidades deverão ser avaliadas e estudadas. Com uma lista de requisitos bem elaborada, e com o auxílio de uma análise de aderência, é possível avaliar se tais tecnologias poderão ser utilizadas para a integração de sistemas legados. Assim, dentro da disciplina de automação, há um vasto caminho a evoluir a fim de levar a informação desde o chão de fábrica até às nuvens computacionais.

O presente trabalho tem como objetivo analisar um ambiente real de chão de fábrica, categorizá-lo, e identificar oportunidades de utilização de sistemas e equipamentos IIoT, observando as tecnologias NB-IoT e LoRaWAN, e avaliando se estas são aderentes ao ambiente objeto deste estudo.

Este trabalho encontra-se organizado como segue. A Seção 2 apresenta as características da Indústria 4.0 e da IIoT. A Seção 3 explica o que são as LPWAN, e sua importância, enquanto a Seção 4 aborda as tecnologias LoRaWAN e NB-IoT. Na Seção 5 é exposto o ambiente avaliado, sendo o estudo de caso tratado na Seção 6. 


\section{Indústria 4.0 vs IIoT}

O termo Indústria 4.0 foi usado primeiramente na Alemanha em 2011, e em 2013 foi apresentado ao governo alemão um relatório com um conjunto de recomendações para a sua implementação [Coelho, 2016], baseando-se em seis princípios:

- Interoperabilidade: habilidade de pessoas e sistemas se conectarem através da computação em nuvem e da IoT.

- Virtualização: sensores interconectados que monitoram processos físicos e permitem modelos de simulação.

- Descentralização: tomada de decisão pelos sistemas sem intervenção humana, com base em regras e parâmetros.

- Capacidade em tempo real: coletar e analisar dados, entregando conhecimento imediatamente.

- Orientação a Serviço: serviços através da computação em nuvem.

- Modularidade: adaptação das fábricas inteligentes através da reposição ou expansão de módulos individuais.

IIoT é usada para definir o conjunto de tecnologias e serviços, permitindo interconectar dispositivos, computadores e objetos inteligentes por meio da internet. Uma fábrica ou processo que só possui a IIoT, não pode ser considerada como pertencente a Indústria 4.0. Ao lado de tecnologias como o Big Data, IoT e computação em nuvem, é possível reduzir custos e tornar a produção mais automatizada e autônoma [Coelho, 2016]. Buscando identificar novas oportunidades, ou revisitando processos carentes de monitoramento, uma análise profunda de todo o ambiente se torna necessária.

\section{Low Power Wide Area Networks (LPWAN)}

LPWAN é um termo genérico para um grupo de tecnologias que permitem comunicações em longa distância, com baixo custo e reduzido consumo de energia [Lin et al., 2017]. Tal tecnologia é adequada para as aplicações IoT que precisam apenas transmitir pequenas quantidades de informações a uma certa distância. O mercado de IoT se expandiu rapidamente e LPWAN teve crescimento rápido. Muitas das tecnologias LPWAN representadas na Figura 1 surgiram em mercados licenciados e não licenciados, como LTE-M, SigFox, LoRa e NB-IoT, dentre outras.

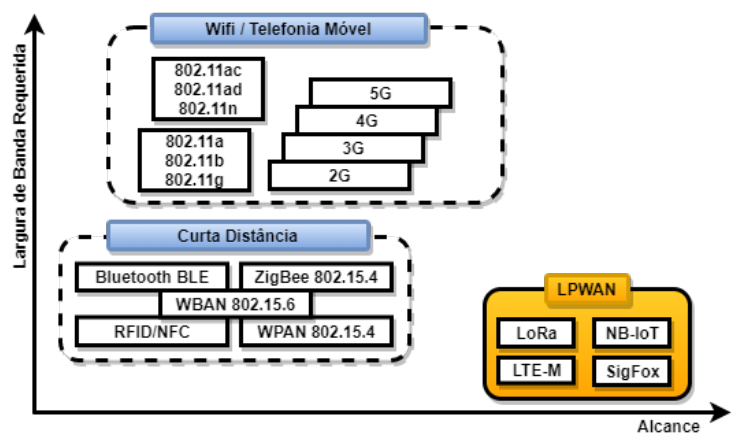

Figura 1. Largura de banda necessária vs. capacidade em curtas distâncias, rede de telefonia celular, e LPWAN. Adaptado de [Sinha et al., 2017]

Segundo [Mekki et al., 2019], a LPWAN pode cobrir distâncias entre 10km e $40 \mathrm{~km}$ em zonas rurais, e entre $1 \mathrm{~km}$ a $5 \mathrm{~km}$ em zonas urbanas, e como já mencionado 
antes, a um baixo custo e baixo consumo energético. Uma importante característica das tecnologias que compõem a LPWAN, é o fato delas trabalharem nas faixas Sub-Ghz, o que impacta diretamente na sua capacidade de transpor obstáculos. Entre estes, Long Range Wide Area Network (LoRaWAN) e Narrowband Internet of Things (NB-IoT) são as duas tecnologias emergentes líderes, que se por um lado possuem diferenças técnicas, por outro, e que em alguns casos, podem ser utilizadas em um mesmo tipo de aplicação.

\section{LoRaWAN e NB-IoT}

A NB-IoT geralmente é usada em ambientes em que a conectividade celular $3 \mathrm{G} / 4 \mathrm{G}$ ou a IEEE 802.11 pode não funcionar de forma satisfatória, como espaços com interferências físicas, estruturas metálicas, ou em grandes áreas rurais. A NB-IoT padroniza três operações quando implantada em outras redes [Ratasuk et al., 2016]: (i) In-band: quando usa blocos de recursos físicos de um prestador de serviço 4G/LTE; (ii) Band Guard: quando regula o espectro não usado em um provedor de serviços de banda de guarda 4G/LTE; e (iii) Independente: quando implantada em um espectro dedicado. A NB-IoT é indicada quando não se precisa transferir consideráveis quantidades de dados e que não exigem baixa latência. Porém, esta faz uso da estrutura LTE, e possui aderência as futuras redes $5 \mathrm{G}$, conforme o $3 \mathrm{GPP}$ e esta é uma vantagem.

Como uma tecnologia LPWAN, esta melhora significativamente o consumo de energia dos dispositivos de IoT e IIoT, podendo a sua bateria ter uma vida útil por volta de 10 anos. Possui também uma eficiência de espectro, especialmente em cobertura interna, por pertencer a uma categoria Sub-Ghz [Mekki et al., 2019]. A Long Range (LoRa) faz parte da LoRa Aliance, também pertence a categoria Sub-Ghz, podendo ser utilizada em uma configuração ponto a ponto, estrela, ou através de conexões com gateways através da rede LoRaWAN. A LoRaWAN é o nome dado ao protocolo que define a arquitetura do sistema, bem como os parâmetros de comunicação usando a tecnologia LoRa. O protocolo LoRaWAN implementa os detalhes de funcionamento, segurança, qualidade do serviço, ajustes de potência visando maximizar a duração da bateria dos módulos, e os tipos de aplicações tanto do lado do módulo quanto do servidor. Assim, pode-se considerar que LoRa é a camada física da rede, e LoRaWAN, que é a camada lógica da rede. Pode-se observar na Tabela 1 as tecnologias LoRaWAN, NB-IoT e outras que estão em evidência na IoT e IIoT.

Tabela 1. Características de tecnologias de loT.

\begin{tabular}{|c|c|c|c|c|c|c|c|c|}
\hline Padrão & IEEE802.11 & BLE & ZigBee Pro & SigFox & LoRaWAN & LTE-M & NB-IoT & 5G \\
\hline Usa 3GPP? & Não & Não & Não & Não & Não & Sim (Release 13) & Sim (Release 13) & Sim (Release 15) \\
\hline Area de Cobertura & $17-30(\mathrm{~m})$ & $\sim 1-50(\mathrm{~m})$ & $\sim 1$ a $250(\mathrm{~m})$ & $<12(\mathrm{~km})$ & $<10(\mathrm{~km})$ & $<10(\mathrm{~km})$ & $<15(\mathrm{~km})$ & $<12(\mathrm{~km})$ \\
\hline $\begin{array}{l}\text { Espectro / } \\
\text { Largura de Banda }\end{array}$ & $\begin{array}{l}2.4 \mathrm{Ghz} \\
(802.11) \\
\end{array}$ & $\begin{array}{c}2.4 \mathrm{Ghz} \\
(802.15 .1)\end{array}$ & $\begin{array}{c}2.4 \mathrm{Ghz} \\
(802.15 .4)\end{array}$ & $900 \mathrm{Mhz}$ & $900 \mathrm{Mhz}$ & 7 - $900 \mathrm{Mhz}$ & 8 - $900 \mathrm{Mhz}$ & $\begin{array}{c}5-900 \mathrm{Mhz} \\
\text { (entre outras) }\end{array}$ \\
\hline $\begin{array}{l}\text { Taxa de } \\
\text { Comunicação }\end{array}$ & $\begin{array}{c}450 \\
(\mathrm{Mbps}) \\
(802.11 \mathrm{n})\end{array}$ & $\begin{array}{c}1 \\
\text { (Mbps) }\end{array}$ & $\begin{array}{c}250 \\
(\mathrm{kbps})\end{array}$ & $\begin{array}{c}\sim 100-600 \\
\quad(\mathrm{bps})\end{array}$ & $\begin{array}{l}\sim 200-50 \\
(\mathrm{kbps})\end{array}$ & $\begin{array}{c}<1 \\
\text { (Mbps) }\end{array}$ & $\begin{array}{l}<144 \\
(\mathrm{kbps})\end{array}$ & $\begin{array}{c}\sim 10 \\
(\mathrm{Gbps})\end{array}$ \\
\hline Custo & $\begin{array}{l}\text { 4.00 USD } \\
\text { (2016) }\end{array}$ & $\begin{array}{l}\text { 4.00 USD } \\
\text { (2016) }\end{array}$ & $\begin{array}{l}3.00 \text { USD } \\
(2016)\end{array}$ & $\begin{array}{c}4.00 \text { USD } \\
(2015) \\
2.64 \text { USD } \\
(2020)\end{array}$ & $\begin{array}{l}\text { 4.00 USD } \\
(2015) \\
2.64 \text { USD } \\
(2020)\end{array}$ & $\begin{array}{c}5.00 \text { USD } \\
(2015) \\
3.30 \text { USD } \\
(2020)\end{array}$ & $\begin{array}{c}4.00 \text { USD } \\
(2015) \\
2-3 \text { USD } \\
(2020)\end{array}$ & $<2.00$ USD \\
\hline Latência & $20-40(\mathrm{~ms})$ & $6(\mathrm{~ms})$ & $40(\mathrm{~ms})$ & $1-30(\mathrm{~s})$ & $61-371(\mathrm{~ms})$ & $50-100(\mathrm{~ms})$ & $1.6-10(\mathrm{~s})$ & $5-50(\mathrm{~ms})$ \\
\hline Segurança & 256 bits & 128 bits AES & 128 bits & 16 bits & 32 bits AES-128 & $128-256$ bits & $128-256$ bits & 256 bits \\
\hline
\end{tabular}

Avaliando os dados contidos na Tabela 1, dentre as diversas tecnologias, quando tem-se longas distâncias para aplicações industriais, já podem ser descartar algumas destas (e.g., IEEE 802.11, BLE e ZigBee) por não atingirem este requisito. Pode-se ex- 
cluir também a rede Sigfox, em função da infraestrutura para se montar uma rede dessas, a baixa capacidade de transmissão na ordem de poucos bytes, alta latência e baixa segurança. A rede LTE-M tem um custo maior de implantação, e um custo maior para se manter; apesar de possuir uma boa taxa de comunicação (pode-se observar estes fatos através da Tabela 1). Como a $5 \mathrm{G}$ ainda não é uma realidade para o Brasil, as melhores opções são as redes LoRaWAN e NB-IoT.

\section{Ambiente Avaliado: ArcelorMittal Vega}

O ambiente de teste é a empresa ArcelorMittal Vega, localizada em São Francisco do Sul/SC, uma das mais modernas unidades de transformação de aços planos do mundo. Com capacidade de produção total de 1,6 milhão de toneladas/ano de bobinas decapadas, laminadas a frio e revestidas por imersão a quente, atende, principalmente, às indústrias automotivas, de eletrodomésticos, de produção de tubos e construção civil.

Esta empresa possui 18 anos de operação, e através dos anos algumas linhas de produção foram modernizadas, expandidas, e até mesmo uma nova linha de galvanização foi adicionada. Desta forma, há um ambiente com uma automação moderna, mas também com itens da época de sua criação. Além disso, a empresa está passando por um projeto de ampliação, que trará um aumento de produção na ordem de $35 \%$, trazendo também duas novas e modernas linhas de produção já com a visão de Indústria 4.0 e digitalização.

\section{Estudo de caso}

Uma planta industrial é composta pela integração de diversos sistemas de automação, e.g., sistemas de medição, máquinas de solda, fornos, etc. Por esta razão, a heterogeneidade faz parte desta realidade. A Tabela 2 elenca e categoriza os principais sistemas de automação industrial, levando em consideração importantes requisitos [Brown et al., 2018], abrangendo os principais sistemas de qualquer planta industrial. Durante a avaliação não foi observada a necessidade da criação de um novo item na Tabela 2.

Tabela 2. Principais requisitos de desempenho em serviços de lloT / automação industrial. Adaptado de [Brown et al., 2018]

\begin{tabular}{|c|c|c|c|c|c|c|}
\hline \multicolumn{2}{|l|}{ Caso de Uso (alto nível) } & Disponibilidade & $\begin{array}{l}\text { Ciclos de } \\
\text { tempo }\end{array}$ & $\begin{array}{l}\text { Tamanho típico do } \\
\text { payload }\end{array}$ & $\begin{array}{l}\text { Número de } \\
\text { dispositivos }\end{array}$ & $\begin{array}{l}\text { Área típica } \\
\text { de serviço }\end{array}$ \\
\hline \multirow{3}{*}{$\begin{array}{l}\text { Controle de } \\
\text { movimento }\end{array}$} & $\begin{array}{l}\text { Impressora } \\
\text { industrial }\end{array}$ & $>99,9999 \%$ & $<2 \mathrm{~ms}$ & 20 bytes & $>100$ & $100 \mathrm{~m} \times 100 \mathrm{mx} 30 \mathrm{~m}$ \\
\hline & $\begin{array}{l}\text { Máquina- } \\
\text { ferramenta }\end{array}$ & $>99,9999 \%$ & $<0,5 \mathrm{~ms}$ & 50 bytes & $\sim 20$ & $15 \mathrm{~m} \times 15 \mathrm{~m} \times 3 \mathrm{~m}$ \\
\hline & $\begin{array}{l}\text { Máquina de } \\
\text { embalagem }\end{array}$ & $>99,9999 \%$ & $<1 \mathrm{~ms}$ & 40 bytes & $\sim 50$ & $10 \mathrm{~m} \times 5 \mathrm{~m} \times 3 \mathrm{~m}$ \\
\hline \multirow[t]{2}{*}{ Robôs móveis } & $\begin{array}{l}\text { Controle de } \\
\text { movimento cooperativo }\end{array}$ & $>99,9999 \%$ & $1 \mathrm{~ms}$ & $40-250$ bytes & 100 & $<1 \mathrm{~km}^{2}$ \\
\hline & $\begin{array}{l}\text { Controle remoto } \\
\text { operado por vídeo }\end{array}$ & $>99,9999 \%$ & $10-100 \mathrm{~ms}$ & $15-150$ bytes & 100 & $<1 \mathrm{~km}^{2}$ \\
\hline \multirow{2}{*}{$\begin{array}{l}\text { Painéis de controles } \\
\text { móveis e com funções } \\
\text { de segurança }\end{array}$} & $\begin{array}{l}\text { Robôs de } \\
\text { montagem } \\
\text { ou fresadoras }\end{array}$ & $>99,9999 \%$ & $4-8 \mathrm{~ms}$ & $40-250$ bytes & 4 & $10 \mathrm{~m} \times 10 \mathrm{~m}$ \\
\hline & Guindastes móveis & $>99,9999 \%$ & $12 \mathrm{~ms}$ & $40-250$ bytes & 2 & $4 \mathrm{~m} \times 60 \mathrm{~m}$ \\
\hline \multicolumn{2}{|c|}{ Automação / Monitoramento de processos) } & $>99,99 \%$ & $>50 \mathrm{~ms}$ & vários & \multicolumn{2}{|c|}{10.000 dispositivos por $\mathrm{km}^{2}$} \\
\hline
\end{tabular}

Durante a fase de análise, foram avaliados os principais sistemas de automação em todas as linhas de produção, incluindo os sistemas considerados como "auxiliares". Todos os dados foram tabulados, e classificados conforme a Tabela 2. Com isso, a Tabela 3 foi elaborada, sendo esta uma amostragem. Outras características também foram avaliadas, e.g., servidores, controladores, remotas, atuadores, existência de banco de dados, etc. 
Tabela 3. Estudo de caso: fração da tabela avaliativa.

\begin{tabular}{|c|c|c|c|c|c|c|}
\hline Nome & Descrição & Fábrica & Rede & Protocolo & Categoria & Sub-Categoria \\
\hline CCK & $\begin{array}{l}\text { Sistema de monitoramento } \\
\text { de energia e utilidades }\end{array}$ & Todas & Ethernet & TCP & \multicolumn{2}{|c|}{$\begin{array}{c}\text { Automação / } \\
\text { Monitoramento de Processos }\end{array}$} \\
\hline Eurotherm & $\begin{array}{l}\text { Sistema de monitoramento } \\
\text { de ponto de orvalho }\end{array}$ & $\begin{array}{l}\text { REC, ENC, } \\
\text { LIR } 1\end{array}$ & Ethernet & Modbus - TCP & \multicolumn{2}{|c|}{$\begin{array}{c}\text { Automação / } \\
\text { Monitoramento de Processos }\end{array}$} \\
\hline Byond & $\begin{array}{l}\text { Sistema de gestão de lingotes } \\
\text { de zinco }\end{array}$ & $\begin{array}{l}\text { GAL } 1 \text { e } \\
\text { GAL } 2\end{array}$ & IEEE 802.11 & $\begin{array}{l}\text { TCP, Zigbee, } \\
\text { MQTT }\end{array}$ & \multicolumn{2}{|c|}{$\begin{array}{c}\text { Automação / } \\
\text { Monitoramento de Processos }\end{array}$} \\
\hline AMPTEM & $\begin{array}{l}\text { Sistema de monitoramento dos } \\
\text { mancais dos cilindros do } \\
\text { laminador de tiras à frio }\end{array}$ & LAM & Ethernet & Zigbee & \multicolumn{2}{|c|}{$\begin{array}{c}\text { Automação / } \\
\text { Monitoramento de Processos }\end{array}$} \\
\hline ViWPD & $\begin{array}{l}\text { Sistema de visão para medição } \\
\text { on-line de largura de chapa }\end{array}$ & GAL 1 & Ethernet & TCP, OPC & \multicolumn{2}{|c|}{$\begin{array}{c}\text { Automação / } \\
\text { Monitoramento de Processos }\end{array}$} \\
\hline SIAS & $\begin{array}{l}\text { Sistema de visão para detecção } \\
\text { on-line de defeitos }\end{array}$ & DEC e GAL 1 & Ethernet & TCP (IP e UDP) & \multicolumn{2}{|c|}{$\begin{array}{c}\text { Automação / } \\
\text { Monitoramento de Processos }\end{array}$} \\
\hline Robô & $\begin{array}{l}\text { Robô estacionário para a } \\
\text { limpeza do pote de Zinco }\end{array}$ & GAL 1 e GAL 2 & Ethernet & $\begin{array}{c}\text { TCP } \\
\text { (IP e UDP), OPC }\end{array}$ & $\begin{array}{l}\text { Controle de } \\
\text { Movimento }\end{array}$ & $\begin{array}{l}\text { Máquina - } \\
\text { Ferramenta }\end{array}$ \\
\hline $\begin{array}{l}\text { Marcadora de } \\
\text { Bobinas }\end{array}$ & $\begin{array}{l}\text { Marcadora de bobinas - } \\
\text { textos alfanuméricos } \\
\text { e código de barras }\end{array}$ & GAL 1 e GAL 2 & Ethernet & TCP (IP e UDP) & $\begin{array}{l}\text { Controle de } \\
\text { Movimento }\end{array}$ & $\begin{array}{l}\text { Impressora } \\
\text { Industrial }\end{array}$ \\
\hline Pontes Rolantes & $\begin{array}{l}\text { Pontes rolantes para } \\
\text { movimentação de } \\
\text { bobinas }\end{array}$ & PIMS & IEEE 802.11 & $\begin{array}{c}\text { OPC, Profibus, } \\
\text { TCP } \\
\text { (IP e UDP), }\end{array}$ & $\begin{array}{l}\text { Painéis de Controles } \\
\text { Móveis e Com } \\
\text { Funções de Segurança }\end{array}$ & $\begin{array}{l}\text { Guindastes } \\
\text { Móveis }\end{array}$ \\
\hline
\end{tabular}

Com o agrupamento (Tabela 3) foi evidenciada que a característica mais predominante são dos sistemas de "Automação / Monitoramento de Processo"; dessa forma, esta foi escolhida para a presente análise. Em termos quantitativos foram catalogados 23 diferentes tipos de sistemas de automação. Sendo que mais de uma linha de produção pode possuir um mesmo sistema. Neste caso, este foi contado como apenas um. Dentro desta categoria, abordando a visão de automação industrial, pode-se destacar como essenciais as seguintes características para os tipos de processos industriais avaliados: (i) Disponibilidade na ordem de 99,99\%; (ii) Ciclo de tempo (também chamado de "scan”), na ordem de 50ms; (iii) Elevado número de dispositivos por $\mathrm{km}^{2}$.

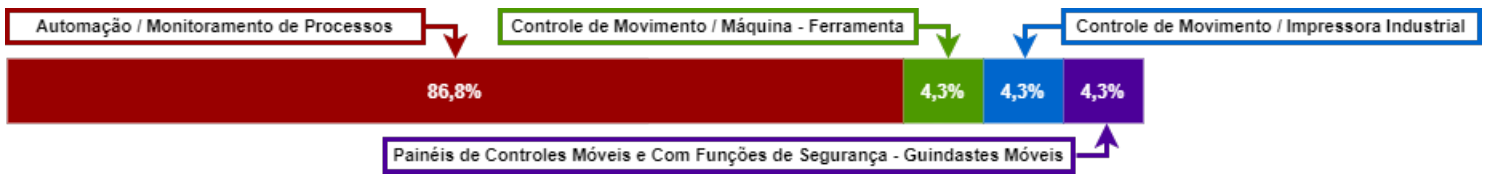

Figura 2. Distribuição observada.

Com a estratificação dos dados contidos na Tabela 3 em sua versão completa, pode-se observar na Figura 2, como a automação / monitoramento de processos se destaca quando comparada com as outras áreas. Além disso, as quatro áreas da Tabela 2 estão representadas. Avaliando os dados tabulados, alguns dos sistemas existentes são aderentes para serem conectados a uma rede utilizando NB-IoT ou LoRaWAN. Mesmo que a quantidade de bytes transmitidos possa parecer pouco, se um desses bytes for utilizado como um "código"para indicar que a mensagem se trata de um equipamento ou instrumento diferente, pode-se transmitir vários dados de diferentes equipamento se forem utilizados escalonadores de processos.

Para a realização de uma "Prova de Conceito"ou Proof of Concept (PoC), a menor infraestrutura para a conexão ponto a ponto da LoRaWAN pode ser a mais indicada. Além do que, existem microcontroladores baseados em ESP32 do tipo System on a Chip (SoC), que já possui a rede LoRa embutida, juntamente com a antena e local para instalação de uma bateria recarregável. Se forem avaliadas áreas com baixo ou nenhum nível de automatização, e.g., pátio de armazenamento de bobinas e almoxarifados em geral, identifica-se dois locais propícios para início de uma PoC. 


\section{Considerações \& Trabalhos futuros}

Com o uso de tecnologias LPWAN como a LoRaWAN e NB-IoT, novas possibilidades são mostradas para a modernização de processos e a entrada na Indústria 4.0. Porém não apenas isso, pois pode significar o barateamento para a automatização de processos em empresas sem um parque minimamente moderno ou tecnológico, principalmente para os casos nos quais a velocidade não seja um forte requisito técnico. Áreas geograficamente distantes, também são fortes candidatos à adoção de uma tecnologia LPWAN. Também pode significar uma maior adoção de tecnologias Software as a Service (SaaS), visto que pequenos dispositivos de IoT e IIoT podem fazer a integração do chão de fábrica até um sistema hospedado em alguma nuvem, e.g., Amazon AWS, Microsoft Azure, Google, etc.

Como continuidade a este trabalho, será realizado o desenvolvimento de um protótipo utilizando LoRaWAN em uma conexão ponto a ponto, para a interconexão de dados de processo no chão de fábrica até um sistema hospedado em um data center, para a avaliação da capacidade transmissão, distância, capacidade de transpor barreiras físicas em uma ambiente com muitas estruturas metálicas. O local escolhido será um dos pátios de expedição de bobinas, por não possuir nenhum sistema de automação.

Agradecimentos: Os autores agradecem o apoio do LabP2D/UDESC e FAPESC.

\section{Referências}

Brown, G. et al. (2018). Ultra-reliable low-latency 5G for industrial automation. Technol. Rep. Qualcomm, 2:52065394.

Coelho, P. M. N. (2016). Rumo à indústria 4.0. PhD thesis, Universidade de Coimbra.

Lin, J., Shen, Z., and Miao, C. (2017). Using blockchain technology to build trust in sharing LoRaWAN IoT. In Proceedings of the 2 nd International Conference on Crowd Science and Engineering, pages 38-43.

Martins, M. R. A. (2015). Análise de convergência para arquiteturas para automação industrial: Abordagem de integração TA e TI. Escola Politécnica da Universidade de São Paulo-USP. Dissertação de Mestrado.

Mekki, K., Bajic, E., Chaxel, F., and Meyer, F. (2019). A comparative study of LPWAN technologies for large-scale iot deployment. ICT express, 5(1):1-7.

Ratasuk, R., Vejlgaard, B., Mangalvedhe, N., and Ghosh, A. (2016). NB-IoT system for M2M communication. In 2016 IEEE wireless communications and networking conference, pages 1-5. IEEE.

Sauter, T., Soucek, S., Kastner, W., and Dietrich, D. (2011). The evolution of factory and building automation. IEEE Industrial Electronics Magazine, 5(3):35-48.

Sinha, R. S., Wei, Y., and Hwang, S.-H. (2017). A survey on LPWA technology: LoRa and NB-IoT. Ict Express, 3(1):14-21.

Strey, V. A., Campos Filho, J. E. d., Santos, M. A. d., Ferreira, C. C., Wiermann, V. V., and Paula, M. A. Q. d. (2017). Desenho e implantação de processos de gestão para automação industrial. In Anais do Seminário de Automação \& TI, pages 140-148.

Wollschlaeger, M., Sauter, T., and Jasperneite, J. (2017). The future of industrial communication: Automation networks in the era of the internet of things and industry 4.0. IEEE industrial electronics magazine, 11(1):17-27. 